\title{
DISCONTINUOUS GALERKIN METHOD FOR STEADY-STATE RICHARDS EQUATION
}

\author{
J.-B. Clément ${ }^{1,2}$, M. Ersoy ${ }^{1}$, F. Golay ${ }^{1}$, D. Sous ${ }^{2,3}$ \\ ${ }^{1}$ Université de Toulon, IMATH, EA 2134, 83957 La Garde, France \\ 2 Université de Toulon, Aix-Marseille Université, CNRS, IRD, Mediterranean Institute of \\ Oceanography (MIO), La Garde, France \\ 3 Univ. Pau \& Pays Adour / E2S UPPA, Laboratoire des Sciences de I'Ingénieur Appliquées \\ à la Mécanique et au Génie Electrique (SIAME) - MIRA, EA4581, 64600, Anglet, France
}

\begin{abstract}
This work is devoted to the numerical simulation of flows in partially saturated porous media. We describe the Richards equation governing the subsurface flow and discuss its range of applicability. A discontinuous Galerkin formulation is used to approximate the steady-state Richards equation. To this end, we present the mathematical framework and a procedure for solving the nonlinear equation. Numerical tests are carried out to highlight properties of the discontinuous Galerkin method and a test case is compared to experimental data to validate the model.
\end{abstract}

Keywords: Discontinuous Galerkin method, Richards equation, nonlinear resolution, unsaturated porous media.

\section{Introduction}

This work enters into a long-term research project aiming to simulate the interactions between waves and groundwater in sandy beaches. Recent studies in laboratory [1, 2] and field studies [3, 4] provided a good experimental understanding of groundwater dynamics and shown a circulation pattern below the swash zone. Up to now, few models have been suggested and their scope remains limited [5, 6]. New numerical developments are necessary to capture a maximum of physical processes, in particular to resolve all along the wave phase the complex dynamics of saturation and pressure fields. The numerical simulation is quite hard to achieve because it requires to solve both nonlinear equations for subsurface water flow and the shallow water equations for surface flow. Additionally, these models has to be coupled in a suitable way in order to respect the physics of the problem. Indeed, on one hand, the dynamics of incoming waves is very fast due to wave run-up and run-down which are covering and uncovering alternatively the beach. But, on the other hand, groundwater circulation is particularly slow [4] because of sediments acting as low-pass filter. Thereby, infiltrations/exfiltrations of the swash zone, sand saturation fluctuations, multiple timescales and various spacescales require a robust and accurate methodology for both numerical schemes and algorithms as well as a careful insight into the modelling.

In the present work, we first focus on the subsurface flow which is assumed to occur in a partially saturated porous medium and can be described by Richards equation. The latter takes into account the actions of gravity (advection) and capillarity (diffusion) but neglecting the flow of the nonwetting phase, namely the air [7]. Richards equation has been widely used to simulate water flow in unsaturated porous media [8-10]. Nevertheless, its derivation is generally rather roughly introduced, which leads us to first set out the main steps to underline some issues in regards to our model problem. Richards equation is a nonlinear parabolic equation but it degenerates into an elliptic equation under saturated conditions. Nonlinearities are due to constitutive laws and, consequently, the solution includes sharp moving fronts with dynamic smooth regions which challenge numerical methods to obtain robust and efficient solutions of Richards equation [9]. Extensive efforts have been made to overcome these difficulties, in particular to find efficient nonlinear solvers for Richards equation [11-13].

Current research into Richards equation consists in advanced spatial and temporal discretizations [9] allowing construction of adaptive approximations in size and/or in order for space and time. A primary focus on spatial discretization can be outlined [9, 10, 14] with the use of finite 
differences, finite volumes, finite elements, mixed finite elements and discontinuous Galerkin. A fundamental step is to tackle this aspect, that is the reason why we consider a steady-state Richards equation. We choose to use discontinuous Galerkin method because it offers suitable advantages for adaptivity and the treatment of flow dynamics [15]. Indeed, discontinuous Galerkin methods may be seen as a method sharing properties both from finite elements and finite volumes : they are based on a variational formulation but in an element-wise fashion. So they are locally conservative which is crucial in fluid dynamics and explains why the method is more and more used for problems in porous media. Moreover, it enables to change the degree of polynomial approximation and to use non-conforming mesh (hanging node). This is an important benefit since it is possible to handle high-order accuracy and hp-adaptation. Various forms of discontinuous Galerkin methods [16] can be used for Richards equation which can be discretized in a primal or mixed formulation. Here, we will focus on primal interior penalty discontinuous Galerkin methods.

The first part of the paper recalls the Richards equation in a comprehensive review along with some classic constitutive laws. Then, a class of primal formulations of discontinuous Galerkin methods are presented as well as linearization methods to solve the nonlinear model problem. The last part of the paper is devoted to the validation of the numerical methods, through the simulation of a water table recharge experiment.

\section{Model problem for unsaturated porous media}

\subsection{Governing equation}

Richards equation is a classic nonlinear parabolic equation to describe flow occurring in the unsaturated zone of an aquifer [7, 9]:

$$
\partial_{t}(\theta(\psi))-\nabla \cdot(\mathbb{K}(\psi) \nabla(\psi+z))=0
$$

where $\psi$ denotes the pressure head $(m), \mathbb{K}$ the tensor of hydraulic conductivity $(m / s), z$ the elevation $(m)$ and $\theta$ the water content.

Richards equation is a widely used model for water flow in unsaturated soils and rocks and thus will be a priori suitable to model groundwater flow in sandy beaches. However, in order to keep in mind its limitations, a synthetic derivation is recalled here. We start with mass conservation principle applied on a two-phase flow in porous medium:

$$
\alpha \in\{\text { air; water }\}, \quad\left\{\begin{array}{l}
\partial_{t}\left(\rho_{\alpha} \Phi S_{\alpha}\right)+\nabla \cdot\left(\rho_{\alpha} \boldsymbol{q}_{\boldsymbol{\alpha}}\right)=0 \\
\boldsymbol{q}_{\boldsymbol{\alpha}}=-\frac{\mathbb{k}_{i} k_{r, \alpha}\left(S_{\alpha}\right)}{\mu_{\alpha}} \nabla\left(p_{\alpha}+\rho_{\alpha} g z\right)
\end{array}\right.
$$

where $\Phi$ denotes the porosity, $S$ the saturation, $\rho_{\alpha}$ the density $\left(k g / m^{3}\right), \boldsymbol{q}_{\boldsymbol{\alpha}}$ the Darcy velocity $(m / s), \mathbb{k}_{i}$ the tensor of intrinsic permeability $\left(m^{2}\right), k_{r, \alpha}$ the relative permeability $\left(m^{2}\right), \mu_{\alpha}$ the dynamic viscosity $(P a \cdot s), p_{\alpha}$ is pressure $(P a)$ and $g$ is gravitational acceleration $\left(\mathrm{m} / \mathrm{s}^{2}\right)$. Here, $\boldsymbol{q}_{\boldsymbol{\alpha}}$ is modelled as an extension of Darcy's law to diphasic system, sometimes called Darcy-Buckingham law. It was initially based on the results of experiments but some theoretical derivations were undertaken via homogenization techniques such as in Whitaker [17]. In any case, underlying assumptions are made about the nature of flows and porous media, in particular, related to Stokes flow.

Additionally, two closure conditions go along with equations (2):

$$
\left\{\begin{array}{l}
S_{\text {air }}+S_{\text {water }}=1, \quad \text { by definition } \\
p_{\text {air }}-p_{\text {water }}=P_{c}\left(S_{\text {water }}\right)
\end{array}\right.
$$

$P_{c}$ is capillary pressure $(P a)$, an invertible function known from experiment. Then, the main hypothesis for Richards equation is used to eliminate the equation for air in Equations (2). Indeed, air viscosity is considered about 55 times smaller than the water viscosity [7] in that case, resulting in the same factor for mobility if relative permeabilities are similar for both fluids. Consequently, pressure gradients balance faster in air than in water phase. Moreover, it is assumed that the air 
phase is connected continuously at every points with the atmosphere, and $p_{a t m}$ is known up to a constant so one can write $p_{a t m}=0$ for convenience and thus:

$$
P_{c}=p_{\text {air }}-p_{\text {water }}=p_{\text {atm }}-p_{\text {water }}=-p_{\text {water }}
$$

Now, for the sake of simplicity, we omit the subscript $\square_{\text {water }}$. Some additional assumptions are usually taken into account that is to say the solid skeleton is not deformable, water density is homogeneous and water is incompressible. This leads to write:

$$
\Phi \partial_{t} S-\nabla \cdot\left(\frac{\mathbb{k}_{i} k_{r}(S)}{\mu} \nabla(p+\rho g z)\right)
$$

By introducing $\theta(S)=\Phi S, \mathbb{K}(S)=\mathbb{k}_{i} k_{r}(S) \frac{\rho g}{\mu}$ and $\psi=\frac{p}{\rho g}$, we recover the original Richards equation (1). It is called the mixed formulation of Richards equation and present better numerical behaviour than water content formulation or head formulation because it is a conservative form, defined for complete saturation and allowing heterogeneous soils [9].

In regards to our situation, Richards equation is a good choice because extensive experience are available in the community especially for its numerical resolution and its coupling with surface flows. Nevertheless, we have to be aware that any effect involving air phase could not be captured such as trapped air pockets. Moreover, fast dynamics due to incoming waves at the boundary for interface condition may cause some troubles with the validity of assumptions taken for DarcyBuckingham law, in particular, the one for Stokes flow.

\subsection{Constitutive laws}

Two constitutive laws, for water content and relative permeability (part of hydraulic conductivity), are needed to close the model problem. They are originally functions of saturation but they are often given in function of pressure head. This substitution is possible given that pressure head is linked by definition to capillary pressure and the latter can be expressed from saturation with (3). These relationships are provided by several models among which Van Genuchten, Brooks and Corey or Haverkamp's relations are the most commonly encountered [8]. They are extensively used even though experiment shows that these relations are actually hysteretic [7, 18] and that their parameters are given with a degree of uncertainty [18].

For Richards equation (1), we express $\mathbb{K}$ and $\theta$ with a classical analytical expression [19]:

$$
(\mathbb{K}(\psi) ; \theta(\psi))= \begin{cases}\left(\frac{K_{s} A}{A+|\psi|^{B}} ; \frac{\theta_{s} C}{C+|\psi|^{D}}\right), & \text { if } \psi \leq 0 \text { (unsaturated zone) } \\ \left(K_{s} ; \theta_{s}\right), & \text { if } \psi>0 \text { (saturated zone) }\end{cases}
$$

where $A, B, C$ and $D$ are empirical parameters, $K_{s}$ is hydraulic conductivity at saturation, $\theta_{s}$ is water content at saturation.

\section{Discontinuous Galerkin approach}

\subsection{Primal formulation}

Steady-state Richards equation (1) is reduced to a non-linear elliptic equation:

$$
-\nabla \cdot(\mathbb{K}(\psi) \nabla(\psi+z))=0
$$

There are many discontinuous Galerkin methods [16] but they can be viewed according to two approaches: primal or mixed formulation. Primal formulation is written as one single equation such as (7) whereas mixed formulation is written as two coupled equations through an intermediate variable such as:

$$
\left\{\begin{array}{l}
\nabla \cdot q=0 \\
q=-\mathbb{K}(\psi) \nabla(\psi+z)
\end{array}\right.
$$


For Richards equation, mixed formulation has been already considered through the Local Discontinuous Galerkin method [20, 21] or SIPG mixed formulation [10]. Here, we consider a primal formulation for Richards equation as matter of interest.

Now, we set some definitions for the discontinuous Galerkin formulation. More careful developments can be found in Dolejší and Feistauer [22] or Rivière [15]. We consider the space $\Omega$ in $\mathbb{R}^{2}$ with $\partial \Omega=\Gamma_{D} \cup \Gamma_{N}$. The model problem is:

$$
\left\{\begin{aligned}
-\nabla \cdot(K \nabla u) & =f, & & \text { in } \Omega \\
u & =g_{D}, & & \text { on } \Gamma_{D} \\
K \nabla u \cdot \boldsymbol{n} & =g_{N}, & & \text { on } \Gamma_{N}
\end{aligned}\right.
$$

$K$ is symmetric, positive definite and bounded, $f$ is in $L^{2}(\Omega), u_{D}$ is in $L^{2}\left(\Gamma_{D}\right)$ and $u_{N}$ is in $L^{2}\left(\Gamma_{N}\right)$. $\Omega$ is subdivided into elements $E$ which forms the tessellation denoted by $\mathcal{E}_{h}$. Elements are made of faces $F . \mathcal{F}_{h}^{I}$ stands for the set of interior faces, $\mathcal{F}_{h}^{D}$ for the set of Dirichlet boundary faces and $\mathcal{F}_{h}^{N}$ for the set of Neumann boundary faces.

Let be two neighbouring elements $E_{l}$ and $E_{r}$ sharing one face $F$. There are two traces of $u$ along $F$ denoted by $u_{l}$ and $u_{r}$. We denote the jump across a face $\llbracket u \rrbracket=u_{l}-u_{r}$, the mean $\{u\}=\frac{1}{2}\left(u_{l}+u_{r}\right)$ and $\boldsymbol{n}$ is the normal vector oriented from $E_{l}$ to $E_{r}$.

The model problem (Eq. (9)) is multiplied by a test function $v$ and integrated on each element $E$. Then, we use Green's theorem and sum all equations to obtain the following variational formulation on the broken Sobolev space $H^{s}\left(\mathcal{E}_{h}\right), s>3 / 2$ :

Find $u \in H^{s}\left(\mathcal{E}_{h}\right)$ such that $\forall v \in H^{s}\left(\mathcal{E}_{h}\right), \quad a_{h}^{\Theta, \sigma_{0}, \sigma_{1}}(u, v)=L_{h}^{\Theta, \sigma_{0}}(v)$ where

$$
\begin{array}{rlr}
a_{h}^{\Theta, \sigma_{0}, \sigma_{1}}(u, v)= & \sum_{E \in \mathcal{E}_{h}} \int_{E} K \nabla u \cdot \nabla v \mathrm{~d} \boldsymbol{x} \\
& -\sum_{F \in \mathcal{F}_{h}^{I}} \int_{F}\left\{K \nabla u \cdot \boldsymbol{n}_{\boldsymbol{F}} \rrbracket \llbracket v \rrbracket \mathrm{d} s\right. & -\sum_{F \in \mathcal{F}_{h}^{D}} \int_{F} K\left(\nabla u \cdot \boldsymbol{n}_{\boldsymbol{F}}\right) v \mathrm{~d} s \\
& -\Theta \sum_{F \in \mathcal{F}_{h}^{I}} \int_{F}\left\{K \nabla v \cdot \boldsymbol{n}_{\boldsymbol{F}} \rrbracket \llbracket u \rrbracket \mathrm{d} s\right. & -\Theta \sum_{F \in \mathcal{F}_{h}^{D}} \int_{F} K\left(\nabla v \cdot \boldsymbol{n}_{\boldsymbol{F}}\right) u \mathrm{~d} s \\
& +\sum_{F \in \mathcal{F}_{h}^{I}} \int_{F} \frac{\sigma_{1}^{F}}{|F|} \llbracket u \rrbracket \llbracket v \rrbracket \mathrm{d} s & +\sum_{F \in \mathcal{F}_{h}^{D}} \int_{F} \frac{\sigma_{0}^{F}}{|F|} u v \mathrm{~d} s \\
L_{h}^{\Theta, \sigma_{0}}(v)= & \sum_{E \in \mathcal{E}_{h}} \int_{E} f v \mathrm{~d} \boldsymbol{x}-\Theta \sum_{F \in \mathcal{F}_{h}^{D}} \int_{F} K\left(\nabla v \cdot \boldsymbol{n}_{\boldsymbol{F}}\right) g_{D} \mathrm{~d} s+\sum_{F \in \mathcal{F}_{h}^{D}} \int_{F} \frac{\sigma_{0}^{F}}{|F|} g_{D} v \mathrm{~d} s \\
& +\sum_{F \in \mathcal{F}_{h}^{N}} \int_{F} K g_{N} v \mathrm{~d} s
\end{array}
$$

$\Theta$ is a parameter to symmetrize the formulation, $\sigma_{0}$ is a boundary penalty parameter to enforce Dirichlet boundary conditions and $\sigma_{1}$ is an interior penalty parameter to penalize jump of the functions and mimic continuity. We can derive several discontinuous Galerkin methods as described in table 1.

\begin{tabular}{|c|c|c|c|c|}
\cline { 3 - 5 } \multicolumn{2}{c|}{} & \multicolumn{3}{c|}{ Symmetrization } \\
\cline { 3 - 5 } \multicolumn{2}{c|}{} & $\Theta=-1$ & $\Theta=0$ & $\Theta=1$ \\
\hline \multirow{2}{*}{ Penalization } & $\sigma=0$ & OBB method & - & global element method \\
\cline { 2 - 5 } & $\sigma \neq 0$ & NIPG & IIPG & SIPG \\
\hline
\end{tabular}

NIPG: Non-symmetric Interior Penalty Galerkin SIPG: Symmetric Interior Penalty Galerkin IIPG: Incomplete Interior Penalty Galerkin OBB method: Oden-Baumann-Babuška method

Table 1: Different types of discontinuous Galerkin methods 
The finite element subspace is taken to be $\mathcal{D}\left(\mathcal{E}_{h}\right)=\left\{v \in L^{2}\left(\Omega: \forall E \in \mathcal{E}_{h},\left.v\right|_{E} \in \mathbb{P}_{p}(E)\right\}\right.$ where $\mathbb{P}_{p}(E)$ denotes the space of polynomials of total degree less than or equal to $p$. Let $N$ be the number of elements and $N_{\text {loc }}$ the local dimension depending on $p$ and the type of reference element. Let $\Phi$ be the global basis functions of the space $\mathcal{D}\left(\mathcal{E}_{h}\right), \varphi$ the local basis functions on each element and $\mathscr{U}$ expansion coefficients. Then, approximation solution $u_{h}$ expands as:

$$
\forall x \in \Omega, \quad u_{h}(x)=\sum_{n=0}^{N-1} \sum_{j=0}^{N_{\text {loc }}} \mathscr{U}_{n}^{j} \Phi_{n}^{j}(x) \text { where } \Phi_{n}^{j}(x)= \begin{cases}\varphi_{n}^{j}(x) & \text { if } x \in E \\ 0 & \text { otherwise }\end{cases}
$$

The choice for values of $\sigma_{0}$ and $\sigma_{1}$ alongside $\Theta$ is crucial because it assures stability of the method among others. One can refer to $[15,16,22,23]$ for a complete analysis of discontinuous Galerkin methods.

\subsection{Linearization techniques}

Richards equation is a nonlinear equation usually solved by an iterative procedure such as fixedpoint iteration or Newton-Raphson method whose choice is determining for computation time performances and convergence.

Studies have been carried out to compare these methods for solving Richards equation [11-13], both for steady-state and transient simulations. They emphasize that fixed-point iteration and even Newton-Raphson's scheme are very sensitive and do not converge systematically according to Richards equation's formulations, initial and boundary conditions.

In this section, the iterative procedure used in the code is described. The variational formulation (10) is written under a matrix system:

$$
K(u) u=f
$$

For Newton-Raphson's solver, residual matrix is defined as $R(u):=K(u) u-f$ and the tangent stiffness matrix by:

$$
K_{t}(u):=\frac{\partial R(u)}{\partial u}=\frac{\partial(K(u) u-f)}{\partial u}=K(u)+\frac{\partial K(u)}{\partial u} u
$$

If we suppose to have obtained at iteration $i-1$ an approximation $u^{i-1}$ of solution $u$ then, at iteration $i$, Newton-Raphson's scheme is:

$$
\left\{\begin{array}{l}
K_{t}\left(u^{i-1}\right) \delta u^{i}=-R\left(u^{i-1}\right) \\
u^{i}=u^{i-1}+\delta u^{i}
\end{array}\right.
$$

The stopping criterion is $\max \left(\frac{\|R(u)\|_{L^{2}(\Omega)}}{\|u\|_{L^{2}(\Omega)}}, \frac{\|\delta u\|_{L^{2}(\Omega)}}{\|K(u) u\|_{L^{2}(\Omega)}}\right)<\varepsilon$ where $\varepsilon$ is a user-defined tolerance and if $u$ or $K(u) u$ do not degenerate.

As example for the construction of $K_{t}$, if $u_{j}$ and $u_{l}$ denote local expansion coefficient (13) for a given element, we are writing its tangent volume local contribution $M_{t}$ which corresponds to the first term of (12):

$$
\begin{aligned}
\left(M_{t}\right)_{1 \leq i, j \leq N_{\mathrm{loc}}} & =\sum_{l} \frac{\partial M_{i l}}{\partial u_{j}} u_{l}=\sum_{l} \frac{\partial}{\partial u_{j}}\left(\int_{E} \mathbb{K}(u) \nabla \varphi_{i} \nabla \varphi_{l} \mathrm{~d} x\right) u_{l}=\sum_{l}\left(\int_{E} \frac{\partial \mathbb{K}(u)}{\partial u_{j}} \nabla \varphi_{i} \nabla \varphi_{l} \mathrm{~d} x\right) u_{l} \\
& =\sum_{l}\left(\int_{E} \frac{\partial \mathbb{K}(u)}{\partial u} \frac{\partial u}{\partial u_{j}} \nabla \varphi_{i} \nabla \varphi_{l} \mathrm{~d} x\right) u_{l}=\sum_{l}\left(\int_{E} \mathbb{K}^{\prime}(u) \varphi_{j} \nabla \varphi_{i} \nabla \varphi_{l} \mathrm{~d} x\right) u_{l} \\
& =\int_{E} \mathbb{K}^{\prime}(u) \varphi_{j}\left(\sum_{l} u_{l} \nabla \varphi_{l}\right) \nabla \varphi_{i} \mathrm{~d} x=\int_{E} \mathbb{K}^{\prime}(u) \varphi_{j} \nabla u \nabla \varphi_{i} \mathrm{~d} x
\end{aligned}
$$

To have a simple fixed-point iteration scheme, the algorithm stays unchanged except that the tangent stiffness matrix defined in (15) is replaced by $K_{t}(u)=\frac{\partial(K(u) u)}{\partial u} \approx K(u)$. 


\section{Numerical validations}

\subsection{One-dimensional convergence results}

We are considering the following system:

$$
\left\{\begin{array}{l}
-\Delta u(x)=\left(4 x^{3}-4 x^{2}-6 x+2\right) \exp \left(-x^{2}\right), \quad \forall x \in(0,1) \\
u(0)=1 \\
u(1)=0
\end{array}\right.
$$

whose the analytical solution is $u(x)=(1-x) \exp \left(-x^{2}\right)$.

We compute numerical error in $L^{2}$ norm and get convergence results for NIPG, SIPG and IIPG methods with linear to cubic polynomial approximations. Penalty values are such that $\sigma_{0}=\sigma_{1}=1$ for NIPG, IIPG and SIPG with $p=3$ but $\sigma_{0}=\sigma_{1}=2$ for SIPG with $p<3$. Uniform meshes of size $h$ are considered.

\begin{tabular}{|c|c|cc|cc|cc|}
\cline { 3 - 8 } \multicolumn{2}{c|}{} & \multicolumn{2}{c|}{ Linear approximation } & \multicolumn{2}{c|}{ Quadratic approximation } & \multicolumn{2}{c|}{ Cubic approximation } \\
\hline \multirow{6}{*}{ Method } & $h$ & $\left\|e_{h}\right\|_{L^{2}(0,1)}$ & Order & $\left\|e_{h}\right\|_{L^{2}(0,1)}$ & Order & $\left\|e_{h}\right\|_{L^{2}(0,1)}$ & Order \\
\hline \multirow{6}{*}{ NIPG } & $1 / 2$ & $9.7104 \mathrm{E}-02$ & - & $1.2899 \mathrm{E}-02$ & - & $6.2398 \mathrm{E}-04$ & - \\
& $1 / 4$ & $2.6019 \mathrm{E}-02$ & 1.8999 & $4.1564 \mathrm{E}-03$ & 1.6339 & $4.2126 \mathrm{E}-05$ & 3.8887 \\
& $1 / 8$ & $6.5796 \mathrm{E}-03$ & 1.9835 & $1.2318 \mathrm{E}-03$ & 1.7545 & $2.7853 \mathrm{E}-06$ & 3.9188 \\
& $1 / 16$ & $1.6361 \mathrm{E}-03$ & 2.0078 & $3.2297 \mathrm{E}-04$ & 1.9314 & $1.7792 \mathrm{E}-07$ & 3.9685 \\
& $1 / 32$ & $4.0652 \mathrm{E}-04$ & 2.0088 & $8.1698 \mathrm{E}-05$ & 1.9830 & $1.1217 \mathrm{E}-08$ & 3.9875 \\
\hline \multirow{6}{*}{ SIPG } & $1 / 2$ & $1.6443 \mathrm{E}-01$ & - & $2.0337 \mathrm{E}-02$ & - & $6.0659 \mathrm{E}-04$ & - \\
& $1 / 4$ & $3.4034 \mathrm{E}-02$ & 2.2724 & $2.4644 \mathrm{E}-03$ & 3.0448 & $2.3031 \mathrm{E}-05$ & 4.7191 \\
& $1 / 8$ & $7.0833 \mathrm{E}-03$ & 2.2645 & $1.7036 \mathrm{E}-04$ & 3.8545 & $1.1354 \mathrm{E}-06$ & 4.3423 \\
& $1 / 16$ & $1.3566 \mathrm{E}-03$ & 2.3844 & $1.4595 \mathrm{E}-05$ & 3.5450 & $5.3777 \mathrm{E}-08$ & 4.4000 \\
& $1 / 32$ & $2.4925 \mathrm{E}-04$ & 2.4444 & $1.2907 \mathrm{E}-06$ & 3.4992 & $2.4664 \mathrm{E}-09$ & 4.4465 \\
\hline \multirow{6}{*}{ IIPG } & $1 / 2$ & $1.37511 \mathrm{E}-01$ & - & $4.4606 \mathrm{E}-02$ & - & $4.4968 \mathrm{E}-03$ & - \\
& $1 / 4$ & $3.53440 \mathrm{E}-02$ & 1.9600 & $7.3239 \mathrm{E}-03$ & 2.6066 & $2.2775 \mathrm{E}-04$ & 4.3034 \\
& $1 / 8$ & $8.67816 \mathrm{E}-03$ & 2.0260 & $1.5210 \mathrm{E}-03$ & 2.2676 & $1.3408 \mathrm{E}-05$ & 4.0863 \\
& $1 / 16$ & $2.11935 \mathrm{E}-03$ & 2.0338 & $3.5286 \mathrm{E}-04$ & 2.1079 & $8.1252 \mathrm{E}-07$ & 4.0445 \\
& $1 / 32$ & $5.20962 \mathrm{E}-04$ & 2.0244 & $8.5108 \mathrm{E}-05$ & 2.0517 & $4.9747 \mathrm{E}-08$ & 4.0297 \\
\hline
\end{tabular}

Table 2: Numerical errors and order of convergence for solution of problem (20)

Convergence rates of primal discontinuous Galerkin method for uniform meshes in one dimension are $[15,22]$ :

\begin{tabular}{|c|l|c|l|}
\hline Method & \multicolumn{1}{|c|}{ NIPG } & SIPG & \multicolumn{1}{c|}{ IIPG } \\
\hline Theoretical order & $\begin{array}{l}p+1 \text { if } p \text { odd } \\
p \text { if } p \text { even }\end{array}$ & $p+1$ & $\begin{array}{l}p+1 \text { if } p \text { odd } \\
p \text { if } p \text { even }\end{array}$ \\
\hline
\end{tabular}

Table 3: Therotical convergence rates for $1 \mathrm{D}$ uniform meshes

Our numerical experiments are in good agreement with theoretical order and with those made in [15] on the same problem. This demonstrates the capability to reach high order approximation using discontinuous Galerkin method.

The code is written to work either from monomial basis or Lagrangian basis functions (classic finite elements basis functions). Results do not differ in both case which is in accordance with discontinuous Galerkin method because basis functions of $\mathcal{D}\left(\mathcal{E}_{h}\right)$ have their support contained in one element. Therefore, any polynomials satisfying a desired orthogonality property to compute basis functions can be chosen. Nevertheless, in order to obtain approximation solution at a particular point, one has to use expansion (13) by computing each coefficient $\mathscr{U}$ and basis functions. Moreover, basis will be chosen to be easily adapted to order of accuracy. 


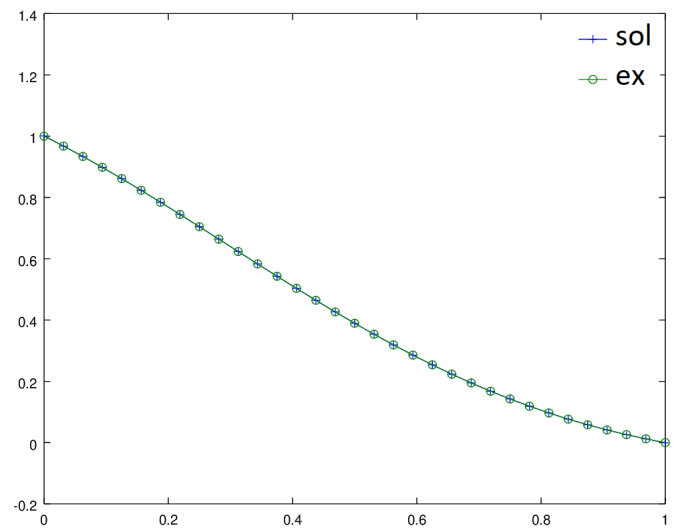

(a) Numerical solution for $\sigma_{0}=\sigma_{1}=2$

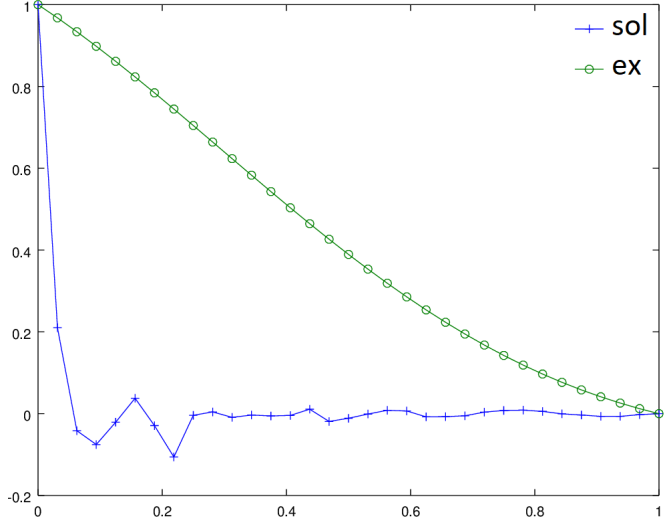

(b) Numerical solution for $\sigma_{0}=\sigma_{1}=10^{15}$

Figure 1: Numerical error for model problem (20) with SIPG method and $p=1$

Numerical results for SIPG method with linear approximation on a grid with 32 elements are presented in figure 1 for two cases.

One observation made during tests is there is a well-known threshold value of penalty values for stability and convergent results. However, it appears that some spurious effects occur for a great penalty values beyond a threshold value such as in figure 1b. This aspect will be tackled in future investigations.

\subsection{Two-dimensional numerical errors}

We are considering the following system, where $(x, y) \in \Omega=[0,1] \times[0,1]$ :

$$
\begin{cases}-\Delta u(x)=8 \pi^{2} \sin (2 \pi x) \sin (2 \pi y) & \text { in } \Omega \\ u=0 & \text { on } \partial \Omega\end{cases}
$$

whose the analytical solution is $u(x, y)=\sin (2 \pi x) \sin (2 \pi y)$.

Here, we focus on the property of discontinuous Galerkin method to treat non-conforming mesh. We focus on SIPG method seeing that there is no difference between the different methods in this case. High order elements could be used but we limit ourselves to linear approximation for brevity. Numerical results are presented for SIPG method with $\sigma_{0}=\sigma_{1}=20$ in the figure 2 .

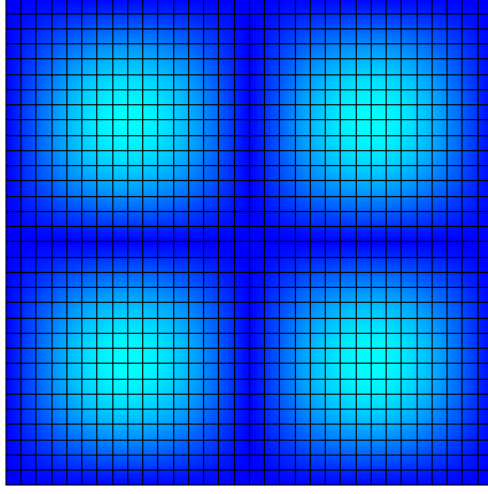

(a) Conforming mesh

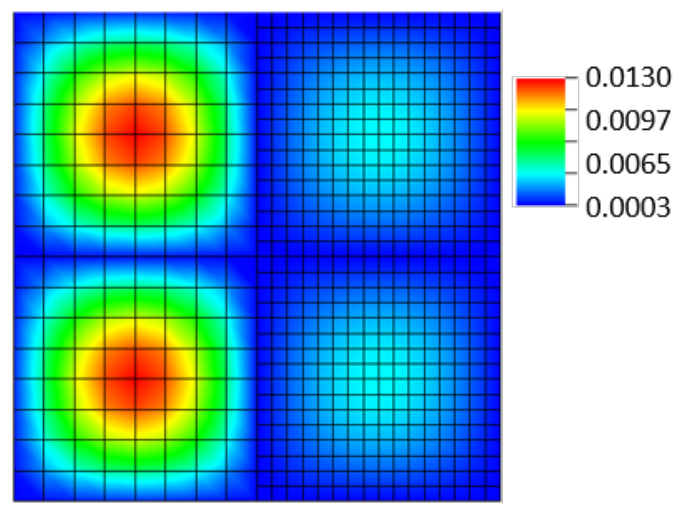

(b) Non-conforming mesh

Figure 2: Numerical error for model problem (21) with SIPG method and $p=1$

First of all, numerical solution is computed on a conforming mesh of 1024 elements on which $\left\|e_{h}\right\|_{\Omega}=1.6085 \times 10^{-3}$ (figure $2 \mathrm{a}$ ). Then, a non-conforming mesh is used. The half part on the 
right is refined. Mesh is made of 696 elements and $\left\|e_{h}\right\|_{\Omega}=3.2272 \times 10^{-3}$ (figure $2 \mathrm{~b}$ ). Numerical comparison with the example given in [22] is in good agreement even though we use a quadrilateral mesh instead of triangles. An interesting point of view about the influence of mesh nature can be found in Wirasaet et al. [24].

We observe that, when mesh is refined with only a difference of refinement level equal to one, discontinuous Galerkin method has good properties with higher numerical errors in the coarser mesh. Hanging nodes do not affect quality of solution. However, a difference of level refinement equal to two makes the solution unstable around hanging nodes. This result is consistent with results obtained in [25] for finite volumes method. In the future, difference of refinement level throughout the mesh should be limited to one. Therefore, we are able to perform mesh adaptation as in $[25,26]$.

\subsection{Comparison with water table recharge experiment}

Water flow through a slab of soil is simulated in Vauclin et al. [19] in which experimental details and results can be found. $\mathbb{K}$ and $\theta$ are taken from (6) with $A=2.99 \times 10^{6}, B=5.0, C=40.000$, $D=2.90, K_{s}=35 \mathrm{~cm} / \mathrm{h}$ and $\theta_{s}=0.30$. We simulate this experiment with the numerical model (7) to obtain the steady-state solution. $h$ stands for hydraulic head $(\mathrm{cm})$ given by $h=\psi+z$. Boundary conditions on the left, at the bottom, on the right for $z>65 \mathrm{~cm}$, on the top for $x>50 \mathrm{~cm}$ are $\nabla h \cdot \boldsymbol{n}=0$. At the top for $x \leq 50 \mathrm{~cm}$, we prescribe a flux $-\mathbb{K}(\psi) \nabla h \cdot \boldsymbol{n}=14.8 \mathrm{~cm} / \mathrm{h}$. On the right for $z \leq 65 \mathrm{~cm}$, the level is kept constant in the ditch: $h=65.0 \mathrm{~cm}$.

Numerical simulation is performed with linear approximation $p=1, \sigma_{0}=\sigma_{1}=20$ on a mesh of 2367 elements. Zones around the expected position of water table and boundary recharge flux are refined. Water table location matches $\psi=0$ and capillary fringe location is established at $\theta(\psi)=0.05 \theta_{s}$. Numerical results are shown in figure 3. Values and red contour lines of hydraulic head $h$ together with distribution of flux are displayed. Calculated water table and capillary fringe are respectively represented by a blue solid line and a blue dotted line. Those of Vauclin et al. are represented by an orange solid line and an orange dotted line.

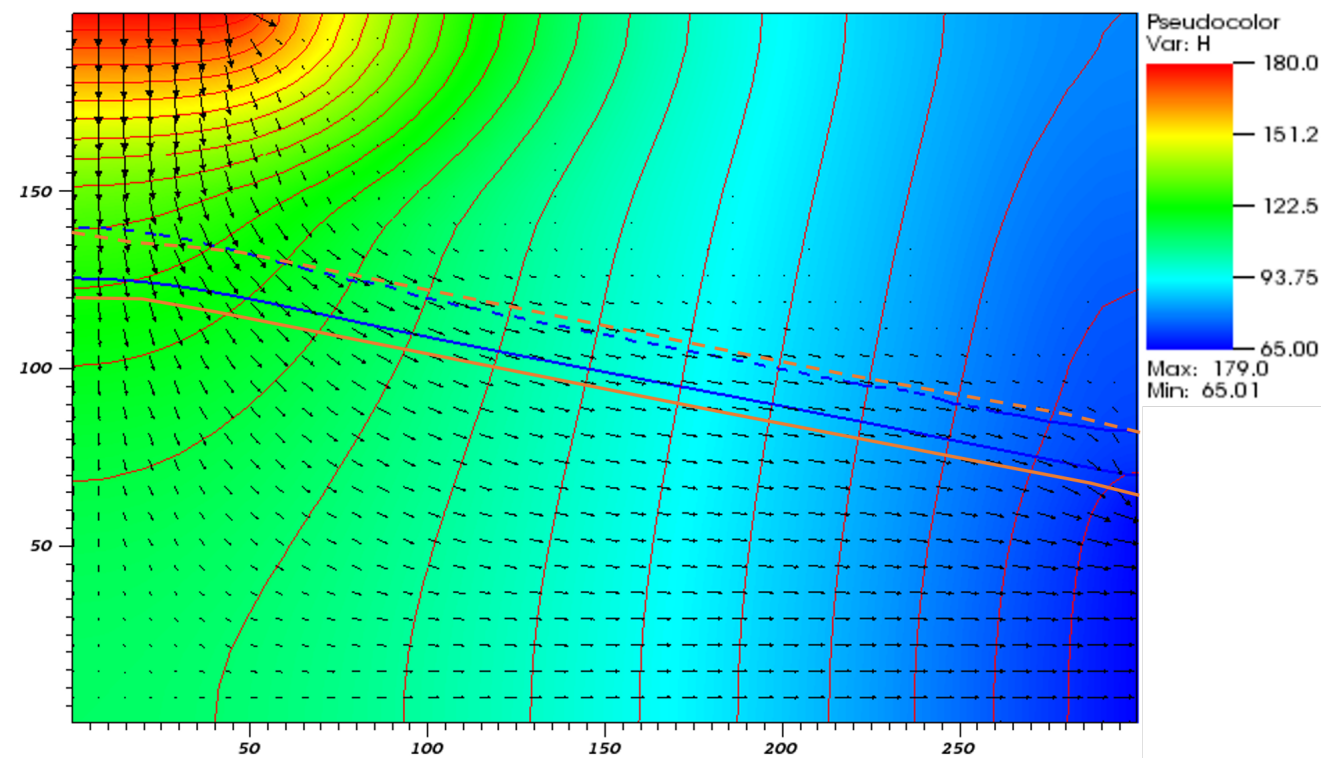

Figure 3: Numerical simulation with distributions flux and hydraulic head $h$

We observe that water table, initially at $z=65 \mathrm{~cm}$, raises because of the recharge which moistens the unsaturated zone. Compared to experiment [19], distribution of flux as well as positions of water table and capillary fringe are in good agreement even though a small shift is to notice. This may be caused by the depth of the seepage boundary condition prescribed by Vauclin et al. above the hydraulic head boundary condition. Distribution of hydraulic head is consistent with experiment within the saturated zone and under the recharge zone. However, on 
the top left-hand area, hydraulic head is higher than one measured and obtained by Vauclin et al. [19]. We will carry out new investigations by increasing polynomial approximation through Adaptive-Mesh-Refinement framework [25, 26].

\section{Conclusion}

In this study, a derivation of Richards equation was undertaken to note some issues which might be debatable if fast dynamics occur, for example, in zones close to the boundary. By choosing a discontinuous Galerkin method, we want to use an easy and efficient hp-adaptation which is one part of the answer, at least numerically. We also consider a Newton-Raphson's method and a fixed-point iteration method to linearise the steady-state Richards equation. Through testcases, we present convergent results which show interesting numerical properties and guidelines for optimization of penalty parameters $\sigma_{0}$ and $\sigma_{1}$. Finally, results agree favourably compared to experimental data of a two-dimensional water table recharge problem even if some features need to be improved.

Further developments are an extension to a time-dependant problem to fully recover the Richards equation and derive some theoretical background modelling to explain fast dynamics at the interface with surface flows.

\section{Acknowledgment}

This work has been supported financially by Provence-Alpes-Côte d'Azur region (France).

\section{References}

[1] Steenhauer, K., Pokrajac, D., Donoghue, T. O. \& Kikkert, G. A.: Subsurface processes generated by bore-driven swash on coarse-grained beaches. Journal of Geophysical Research, vol. 116, no. C4: (2011).

[2] Turner, I. L., Rau, G. C., Austin, M. J. \& Andersen, M. S.: Groundwater fluxes and flow paths within coastal barriers: Observations from a large-scale laboratory experiment (BARDEX II). Coastal Engineering, vol. 113: (2016) pp. 104-116.

[3] Heiss, J. W., Puleo, J. A., Ullman, W. J. \& Michael, H. A.: Coupled surface-subsurface hydrologic measurements reveal infiltration, recharge, and discharge dynamics across the swash zone of a sandy beach. Water Resources Research, vol. 51, no. 11: (2015) pp. 8834-8853.

[4] Sous, D., Petitjean, L., Bouchette, F., Rey, V., Meulé, S., Sabatier, F. \& Martins, K.: Field evidence of swash groundwater circulation in the microtidal rousty beach, france. Advances in Water Resources, vol. 97: (2016) pp. 144-155.

[5] Li, L., Barry, D., Pattiaratchi, C. \& Masselink, G.: BeachWin: modelling groundwater effects on swash sediment transport and beach profile changes. Environmental Modelling \& Software, vol. 17, no. 3: (2002) pp. 313-320.

[6] Malott, S., O'Carroll, D. M. \& Robinson, C. E.: Dynamic groundwater flows and geochemistry in a sandy nearshore aquifer over a wave event. Water Resources Research, vol. 52, no. 7: (2016) pp. 5248-5264.

[7] Szymkiewicz, A.: Modelling Water Flow in Unsaturated Porous Media. Springer Berlin Heidelberg: (2013).

[8] Belfort, B., Younes, A., Fahs, M. \& Lehmann, F.: On equivalent hydraulic conductivity for oscillation-free solutions of richard's equation. Journal of Hydrology, vol. 505: (2013) pp. 202217.

[9] Farthing, M. W. \& Ogden, F. L.: Numerical solution of richards' equation: A review of advances and challenges. Soil Science Society of America Journal, vol. 81, no. 6: (2017) pp. 1257-1269. 
[10] Sochala, P., Ern, A. \& Piperno, S.: Mass conservative BDF-discontinuous galerkin/explicit finite volume schemes for coupling subsurface and overland flows. Computer Methods in Applied Mechanics and Engineering, vol. 198, no. 27-29: (2009) pp. 2122-2136.

[11] Lehmann, F. \& Ackerer, P.: Comparison of iterative methods for improved solutions of the fluid flow equation in partially saturated porous media. Transport in Porous Media, vol. 31, no. 3: (1998) pp. 275-292.

[12] List, F. \& Radu, F. A.: A study on iterative methods for solving richards' equation. Computational Geosciences, vol. 20, no. 2: (2016) pp. 341-353.

[13] Paniconi, C. \& Putti, M.: A comparison of picard and newton iteration in the numerical solution of multidimensional variably saturated flow problems. Water Resources Research, vol. 30, no. 12: (1994) pp. 3357-3374.

[14] Baron, V., Coudière, Y. \& Sochala, P.: Adaptive multistep time discretization and linearization based on a posteriori error estimates for the richards equation. Applied Numerical Mathematics, vol. 112: (2017) pp. 104-125.

[15] Rivière, B.: Discontinuous Galerkin Methods for Solving Elliptic and Parabolic Equations. Society for Industrial and Applied Mathematics: (2008).

[16] Arnold, D. N., Brezzi, F., Cockburn, B. \& Marini, L. D.: Unified analysis of discontinuous galerkin methods for elliptic problems. SIAM Journal on Numerical Analysis, vol. 39, no. 5: (2002) pp. 1749-1779.

[17] Whitaker, S.: Flow in porous media i: A theoretical derivation of darcy's law. Transport in Porous Media, vol. 1, no. 1: (1986) pp. 3-25.

[18] Gray, W. G. \& Hassanizadeh, S. M.: Paradoxes and realities in unsaturated flow theory. Water Resources Research, vol. 27, no. 8: (1991) pp. 1847-1854.

[19] Vauclin, M., Khanji, D. \& Vachaud, G.: Experimental and numerical study of a transient, twodimensional unsaturated-saturated water table recharge problem. Water Resources Research, vol. 15, no. 5: (1979) pp. 1089-1101.

[20] Li, H., Farthing, M., Dawson, C. \& Miller, C.: Local discontinuous galerkin approximations to richards' equation. Advances in Water Resources, vol. 30, no. 3: (2007) pp. 555-575.

[21] Li, H., Farthing, M. \& Miller, C.: Adaptive local discontinuous galerkin approximation to richards' equation. Advances in Water Resources, vol. 30, no. 9: (2007) pp. 1883-1901.

[22] Dolejší, V. \& Feistauer, M.: Discontinuous Galerkin Method. Springer International Publishing: (2015).

[23] Epshteyn, Y. \& Rivière, B.: Estimation of penalty parameters for symmetric interior penalty galerkin methods. Journal of Computational and Applied Mathematics, vol. 206, no. 2: (2007) pp. 843-872.

[24] Wirasaet, D., Tanaka, S., Kubatko, E. J., Westerink, J. J. \& Dawson, C.: A performance comparison of nodal discontinuous galerkin methods on triangles and quadrilaterals. International Journal for Numerical Methods in Fluids, vol. 64, no. 10-12: (2010) pp. 1336-1362.

[25] Ersoy, M., Golay, F. \& Yushchenko, L.: Adaptive multiscale scheme based on numerical density of entropy production for conservation laws. Open Mathematics, vol. 11, no. 8: (2013).

[26] Golay, F., Ersoy, M., Yushchenko, L. \& Sous, D.: Block-based adaptive mesh refinement scheme using numerical density of entropy production for three-dimensional two-fluid flows. International Journal of Computational Fluid Dynamics, vol. 29, no. 1: (2015) pp. 67-81. 